\title{
Toll-Like Receptor 4/Spleen Tyrosine Kinase Complex in High Glucose Signal Transduction of Proximal Tubular Epithelial Cells
}

\author{
Won Seok Yang ${ }^{\mathrm{a}}$ Joon-Seok Kim ${ }^{\mathrm{a}}$ Nam Jeong Han ${ }^{\mathrm{b}}$ Mee Jeong Lee ${ }^{\mathrm{c}}$ Su-Kil Park \\ aDivision of Nephrology, Department of Internal Medicine, Asan Medical Center, College of Medicine, \\ University of Ulsan, Seoul, Korea, ${ }^{b}$ Asan Institute for Life Sciences, Seoul, Korea, 'Department of \\ Pediatrics, College of Medicine, Dankook University, Cheonan, Korea
}

\section{Key Words}

High glucose $\cdot$ High-mobility group box-1 - MyD88 - Spleen tyrosine kinase $\cdot$ Toll-like receptor $4 \cdot$ Transforming growth factor- $\beta 1$

\begin{abstract}
Background/Aims: High glucose activates spleen tyrosine kinase (Syk) in human proximal tubular epithelial cells (HK-2 cells), which leads to NF-KB activation and transforming growth factor- $\beta 1$ (TGF- $\beta 1$ ) production. We explored the signal transduction pathway from high glucose to Syk activation. Methods: The pathway was evaluated by siRNA transfection, immunoprecipitation and Western blot. Results: High glucose stimulated Syk activation within $10 \mathrm{~min}$. Depletion of toll-like receptor 4 (TLR4) attenuated high glucose-induced Syk activation, NF-KB p65 nuclear translocation, and TGF- $\beta 1$ production. In addition, TLR4 inhibitor (CLI-095), TLR4-neutralizing antibody, and depletion of myeloid differentiation factor 88 (MyD88) all attenuated high glucose-induced Syk activation. As an evidence of TLR4 activation, interleukin-1 receptor-associated kinase 1 was recruited to MyD88 and TLR4 upon exposure to high glucose. Syk was co-immunoprecipitated with TLR4, and Syk bound to TLR4 was activated by high glucose. High-mobility group box-1 (HMGB-1), an endogenous activator of TLR4, rapidly increased in TLR4 immunoprecipitates upon high glucose stimulation, and this association was reduced by $\mathrm{N}$-acetylcysteine, an antioxidant. An HMGB-1 inhibitor glycyrrhizin suppressed high glucose-induced Syk activation. Conclusion: Syk is constitutively associated with TLR4. High glucose induces an immediate, reactive oxygen species-dependent, extracellular release of HMGB-1 which binds to TLR4 and activates it, leading to Syk activation.
\end{abstract}

Copyright $(2015$ S. Karger AG, Basel

Su-Kil Park, M.D.

KARGER 125
Department of Internal Medicine, Asan Medical Center, University of Ulsan, 88 Olympic-Ro 43-Gil, SongPa-Gu, Seoul 138-736 (Korea)

Tel. +82230103263, Fax +82230106963, E-Mail skpark@amc.seoul.kr 


\section{Introduction}

Spleen tyrosine kinase (Syk) is a non-receptor protein tyrosine kinase, which transmits B-cell antigen receptor or Fc-receptor signaling of hematopoietic cells including mast cells, lymphocytes, neutrophils and monocytes, and thereby regulates the immune response [1]. In chronic inflammatory diseases including allergic asthma and rheumatoid arthritis, Syk plays a crucial role in the pathogenesis and has become a new promising therapeutic target [2].

Besides the hematopoietic cells, Syk is widely distributed in many other types of cells [3]. Our previous studies suggested that Syk might be implicated in the diabetic kidney disease. In cultured human glomerular endothelial cells [4], we found that high glucose rapidly activates Syk, which leads to tyrosine phosphorylation of $I \kappa B \alpha$ and thereby activates nuclear factor $-\kappa B(N F-\kappa B)$, suggesting that Syk could be an important mediator in the intracellular signal transduction for high glucose-induced cytokine production that is dependent on NF$\kappa \mathrm{B}$ activation, including chemokine (C-C motif) ligand 2 (CCL2). Similarly, in human proximal tubular epithelial cells [5], high glucose activated Syk, which in turn resulted in activations of activator protein- 1 (AP- 1 ) and NF- $\mathrm{kB}$, leading to transforming growth factor- $\beta 1$ (TGF- $\beta 1$ ) gene expression. As such, Syk plays an important role in high glucose signal transduction, but it remains unknown how high glucose stimulates Syk activation.

Toll-like receptors (TLRs) recognize pathogen-associated molecular patterns that are expressed on infectious agents such as lipopolysaccharides (LPS) in Gram-negative bacteria, and mediate the production of cytokines necessary for the development of effective immunity [6]. In neutrophils [7], monocytes [8] and macrophages [9], Syk is associated with toll-like receptor 4 (TLR4), and plays a key role in the signal transduction of TLR4 that is activated by LPS. In addition to binding exogenous ligands derived from pathogens, TLRs interact with endogenous molecules released from damaged tissues and regulate many sterile inflammation processes [10]. Recent studies have shown that TLR4 is also implicated in the pathogenesis of diabetic nephropathy [11-14]. High glucose stimulated TLR4 gene transcription and thereby its protein expression in tubular epithelial cells and mesangial cells, while TLR4 antagonist or deficiency of TLR4 had protective effects on diabetic nephropathy. Though high glucose was shown to induce TLR4 expression, it is not known whether TLR4 directly participates in high glucose signal transduction and regulates Syk activation before its expression is increased.

In the present study, therefore, we explored the signal pathway from high glucose to Syk activation by investigating the interaction between TLR4 and Syk in human proximal tubular epithelial cells (HK-2 cells).

\section{Materials and Methods}

\section{Materials}

D-glucose and glycyrrhizin were purchased from Sigma-Aldrich (St. Louis, MO, USA). Protein A/GPLUS Agarose and antibodies to human TLR4 (for immunoprecipitation, HTA-125 that detects TLR4/ myeloid differentiation factor 2 complex; for immunoblot, $\mathrm{H}-80$ that detects TLR4), myeloid differentiation factor 88 (MyD88), interleukin-1 receptor-associated kinase 1 (IRAK-1), high-mobility group box-1 (HMGB1), p65, Syk, TGF- $\beta 1$, actin, and histone H3 were from Santa Cruz Biotechnology (Santa Cruz, CA, USA). Antibody to human phospho-Syk (pY525) was purchased from Epitomics (clone EP575(2)Y, Burlingame, CA, USA). Neutralizing anti-TLR4 and control IgG antibodies were from eBioscience (San Diego, CA, USA). TLR4-siRNA, MyD88-siRNA and control-siRNAs were from Life Technologies (Ambion ${ }^{\circledR}$; Seoul, Korea). CLI095 was from InvivoGen (San Diego, CA, USA).

\section{Cell culture and treatment}

HK-2 cells were obtained from the American Type Culture Collection (Manassas, VA, USA). HK-2 cells are human renal proximal tubular epithelial cells which are immortalized by transduction with human 


\section{Cellular Physiology Cell Physiol Biochem 2015;35:2309-2319 \begin{tabular}{l|l} 
DOI: 10.1159/000374034 & $\begin{array}{l}\text { O 2015 S. Karger AG, Basel } \\
\text { www.karger.com/cpb }\end{array}$ \\
\hline
\end{tabular} \\ Yang et al.: TLR4/Syk in High Glucose Signal Transduction}

papilloma virus 16 E6/E7 genes. Cells were cultured in RPMI media (Life Technologies) supplemented with $10 \%$ fetal bovine serum (FBS; Gibco ${ }^{\circledR}$, Life Technologies). Before each experiment, the cells were growtharrested for $72 \mathrm{~h}$ in serum-free DMEM media (Life Technologies) containing $5.5 \mathrm{mM}$ glucose. The $72 \mathrm{~h}$ rest was adopted to make the experimental condition same with our previous study [5], in which we tested the effect of high glucose on TGF- $\beta 1 \mathrm{mRNA}$ and protein expression. It required a time for TGF- $\beta 1 \mathrm{mRNA}$ of the cells cultured in the media containing FBS to decline to basal level after deprivation of FBS, and the stimulatory effect of high glucose on TGF- $\beta 1$ mRNA was most consistent after $72 \mathrm{~h}$ starvation. To determine the signal transduction pathway from high glucose to Syk activation, HK-2 cells were treated with 5.5 or 30 $\mathrm{mM}$ glucose for different times, depending on the signal molecules to be evaluated. In the experiments using signal inhibitors including CLI-095 and N-acetylcysteine, the cells were preincubated with the inhibitors for $30 \mathrm{~min}$ before high glucose stimulation. In case of glycyrrhizin, it was added simultaneously with high glucose stimulation.

\section{Transfection of siRNA}

Cells $\left(1 \times 10^{5}\right.$ cells $)$ seeded in a 6 -well plate and cultured for $24 \mathrm{~h}$ were transfected with TLR4-siRNA, MyD88-siRNA or a nonspecific, scrambled, control-siRNA using Lipofectamine ${ }^{\circledR}$ Reagent (Life Technologies). In brief, 100 pmol siRNAs were mixed and incubated with $10 \mu \mathrm{l}$ of lipofectamine reagent diluted in Opti$\mathrm{MEM}^{\circledR}$ medium (Life Technologies) without antibiotics for 15 minutes at room temperature to allow siRNAlipofectamine complexes to form. The siRNA-lipofectamine complexes were added to each culture dish containing fresh medium without serum, and incubated at $37{ }^{\circ} \mathrm{C}$ in a $\mathrm{CO}_{2}$ incubator for $6 \mathrm{~h}$. Thereafter, the medium was replaced with complete growth medium containing serum, and the cells were further incubated for $18 \mathrm{~h}$. After then, the cells were placed in serum-free DMEM media (glucose $5.5 \mathrm{mM}$ ) for $72 \mathrm{~h}$ and subjected to the experiment.

\section{Western blot analysis}

To obtain whole cell lysate, the treated cells were washed with phosphate buffered saline (PBS), and lysed on ice for $10 \mathrm{~min}$ in $100 \mu \mathrm{l}$ of lysis buffer containing $50 \mathrm{mM}$ Tris- $\mathrm{HCl}$ (pH 7.4), $150 \mathrm{mM} \mathrm{NaCl}, 1 \% \mathrm{NP}-40,0.25 \%$ sodium deoxycholate, protease and phosphatase inhibitors. After centrifugation for 20 minutes at $4^{\circ} \mathrm{C}(10,000$ $g$ ), the supernatant was obtained as whole cell lysate. To obtain nuclear protein, the treated cells were at first treated with lysis buffer A (25 mM Hepes ( $\mathrm{pH} 7.9$ ), $50 \mathrm{mM} \mathrm{KCl}, 0.5 \%$ Igepal CA-630, protease and phosphatase inhibitors) on ice for $10 \mathrm{~min}$. The cell lysates were centrifuged for 5 minutes at $4^{\circ} \mathrm{C}(800 \times g)$ and the supernatant (cytosolic fraction) was removed. The pellet was washed twice with washing buffer (25 mM Hepes (pH 7.9), $50 \mathrm{mM} \mathrm{KCl,} \mathrm{protease} \mathrm{and} \mathrm{phosphatase} \mathrm{inhibitors)} \mathrm{and} \mathrm{centrifugation} \mathrm{for} 5$ minutes at $4^{\circ} \mathrm{C}(800 \times g)$. The remaining pellet was lysed again with lysis buffer B $(25 \mathrm{mM}$ Hepes (pH 7.9), $500 \mathrm{mM}$ $\mathrm{KCl}, 10 \%$ glycerol, protease and phosphatase inhibitors) and subjected to repeated freezing and thawing with liquid nitrogen and a water bath set to $37{ }^{\circ} \mathrm{C}$. Thereafter, the nuclear lysates were incubated at $4{ }^{\circ} \mathrm{C}$ for $20 \mathrm{~min}$ on a rocking platform. Finally, nuclear protein was obtained after centrifugation for 20 minutes at $4^{\circ} \mathrm{C}(10,000 \times g)$. Equal amounts of whole cell lysates or nuclear proteins were separated by sodium dodecyl sulfate-polyacrylamide gel electrophoresis on $9 \sim 15 \%$ gels and transferred to an Immobilon-P membrane (Millipore, Bedford, MA, USA). The membrane was probed with the respective primary antibody. Bands were visualized using horseradish peroxidase conjugated secondary antibodies and the enhanced chemiluminescence agent (Luminata ${ }^{\mathrm{TM}}$ Forte Western HRP Substrate; Millipore).

\section{Immunoprecipitation}

The treated cells were lysed on ice as in Western blot, and whole cell lysates were obtained. The cell lysates $(500 \mu \mathrm{g})$ were incubated with $5 \mu \mathrm{g}$ of antibody to TLR4 (HTA-125) or MyD88 for $2 \mathrm{~h}$ at $4{ }^{\circ} \mathrm{C}$, and then incubated with protein $\mathrm{A} / \mathrm{G}$-agarose beads at $4^{\circ} \mathrm{C}$ under rotary agitation for $16 \mathrm{~h}$. After washing with PBS, the protein A/G-agarose beads were recovered after centrifugation for 5 minutes at $4{ }^{\circ} \mathrm{C}(10,000 \times \mathrm{g})$, and resuspended in sample buffer, boiled for $10 \mathrm{~min}$, and analyzed by Western blotting.

\section{Statistical analysis}

Data are presented as means \pm SE (standard error), with $n$ representing the number of different experiments. An analysis of variance with Dunnett multiple-comparisons test was used to determine statistically significant differences between groups. A p value of $<0.05$ was considered statistically significant. 


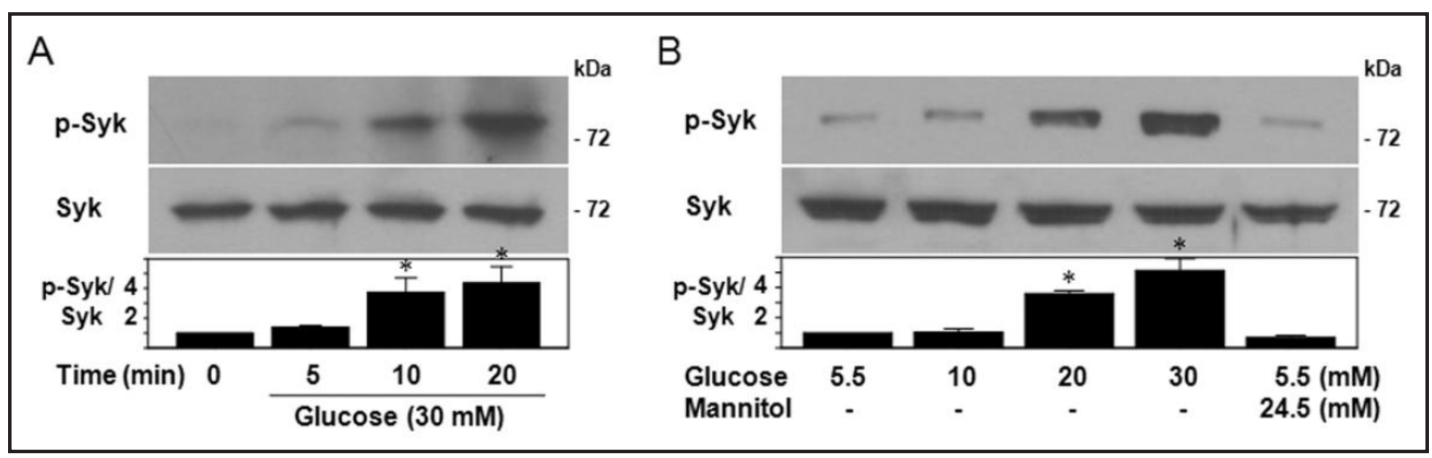

Fig. 1. High glucose rapidly activates Syk in HK-2 cells. (A) HK-2 cells were incubated with 30 mM glucose for $0,5,10$ or $20 \mathrm{~min}(\mathrm{n}=5)$. In another experiment (B), the cells were incubated with different concentrations of glucose or mannitol (as an osmotic control) for $10 \mathrm{~min}(\mathrm{n}=3)$. Whole-cell lysates were immunoblotted with an anti-phospho-Syk antibody. Thereafter, the membranes were stripped and reprobed with an anti-Syk antibody. ( ${ }^{*} \mathrm{p}<0.05$ compared with control).

\section{Results}

High glucose rapidly activates Syk

In our previous study, high glucose activated Syk, which led to activation of NF- $\kappa B$ and increased TGF- $\beta 1$ production [5]. Phosphorylation of tyrosines 525 and 526 of human Syk, which are located in the activation loop of the Syk kinase domain, is essential for its function [15]. Consistent with our previous study, high glucose increased Tyr525 phosphorylation of Syk within $10 \mathrm{~min}$ in a time- and concentration-dependent manner, while mannitol did not (Fig. 1).

Depletion of TLR4 attenuates high glucose-induced Syk activation, NF- «B p65 nuclear translocation, and TGF- $\beta 1$ production

To explore whether TLR4 mediates high glucose-induced Syk activation, we transfected HK-2 cells with control-siRNA or TLR4-siRNA, and incubated them with 5.5 or $30 \mathrm{mM}$ glucose for $10 \mathrm{~min}$ and then assessed Syk activation in the immunoblot of whole cell lysates. Depletion of TLR4 by transfection of TLR4-siRNA abolished high glucose-induced Syk activation, while control-siRNA did not (Fig. 2A).

The major form of NF- $\mathrm{KB}$ is composed of a dimer of p50 and p65 subunits, and is sequestered in the cytoplasm through its tight association with specific inhibitory proteins

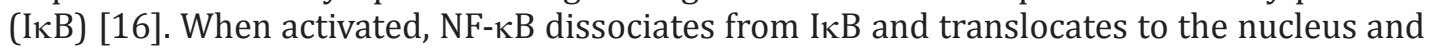
binds to a specific sequence in DNA, which in turn results in gene transcription. To assess the effect of specific depletion of TLR4 on nuclear translocation of p65 protein, we transfected control-siRNA or TLR4-siRNA into the cells and then incubated the cells with 5.5 or $30 \mathrm{mM}$ glucose for $30 \mathrm{~min}$. The amount of p65 in nuclear protein was analyzed by Western blot. As shown in Fig. 2B, TLR4-siRNA abolished high glucose-induced p65 nuclear translocation.

To evaluate the effect of depletion of TLR4 on high glucose-induced TGF- $\beta 1$ production, we transfected HK-2 cells with control-siRNA or TLR4-siRNA, and then incubated them with 5.5 or $30 \mathrm{mM}$ glucose for $24 \mathrm{~h}$. Western blot analysis of whole cell lysates was performed using ant-TGF- $\beta 1$ antibody. High glucose significantly increased total amount of TGF- $\beta 1$ protein as compared with control. By contrast, depletion of TLR4 by transfection of TLR4siRNA abolished high glucose-induced TGF- $\beta 1$ production (Fig. $2 \mathrm{C}$ ).

\section{Inhibition of TLR4 attenuates high glucose-induced Syk activation}

CLI-095, also known as TAK-242, selectively binds to TLR4 and interferes with the interactions between TLR 4 and its adaptor molecules, TIRAP and TRAM [17]. We evaluated the effect of TLR4 inhibitor on high glucose-induced Syk activation. As shown in Fig. 3A, phosphorylation of Syk was increased by high glucose, while this increase was attenuated by CLI-095.

\section{KARGER}



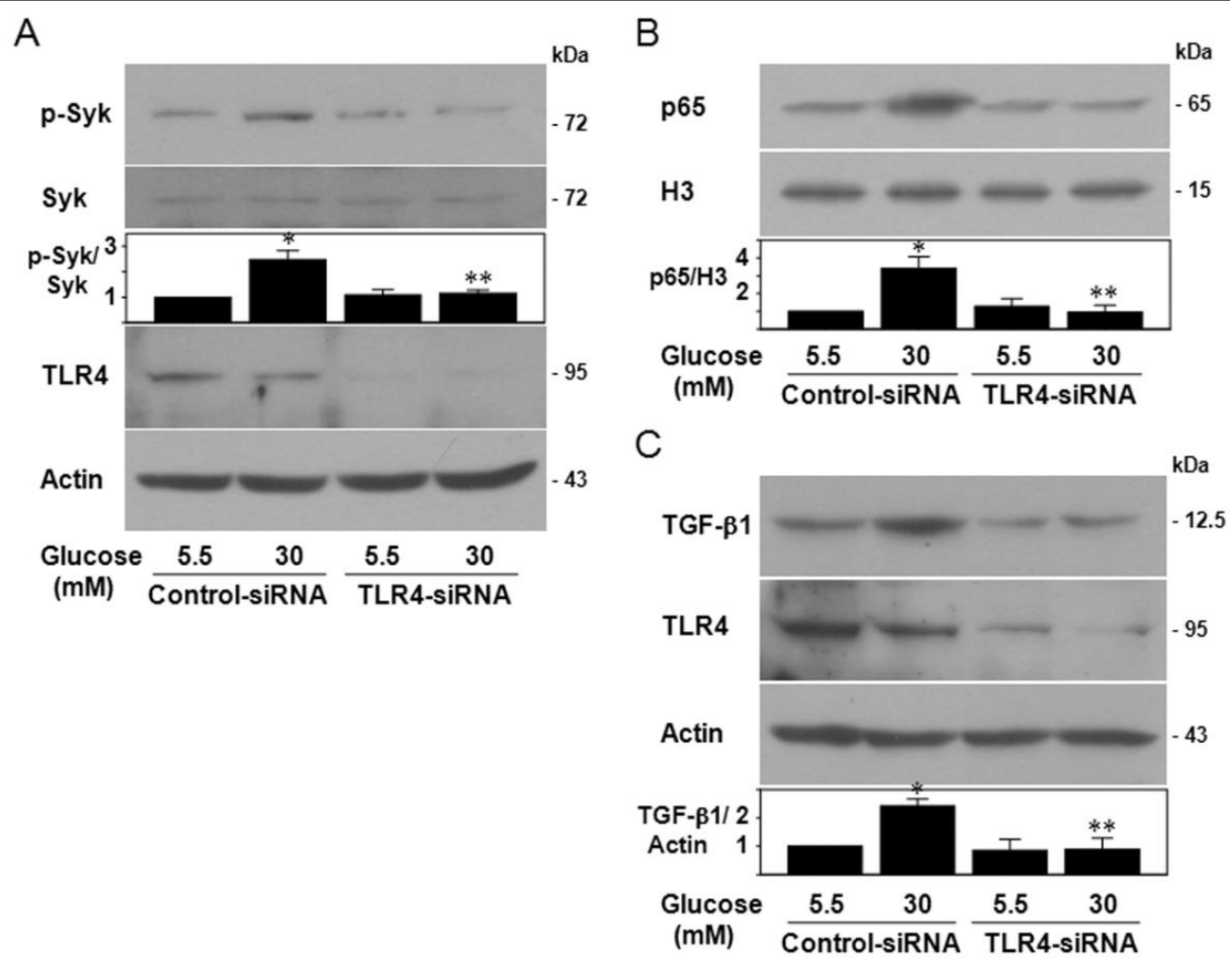

Fig. 2. Depletion of TLR4 attenuates high glucose-induced Syk activation, NF- $\kappa B$ p65 nuclear translocation, and TGF- $\beta 1$ production. (A) HK-2 cells were transfected with control-siRNA or TLR4-siRNA, and then incubated with 5.5 or $30 \mathrm{mM}$ glucose for $10 \mathrm{~min}(\mathrm{n}=3)$. Whole-cell lysates were immunoblotted with an anti-phospho-Syk antibody. Thereafter, the membranes were stripped and reprobed with an anti-Syk, antiTLR4 or anti-actin antibody. ( ${ }^{*} \mathrm{p}<0.05$ compared with control, ${ }^{* *} \mathrm{p}<0.05$ as compared with control-siRNA and $30 \mathrm{mM}$ glucose) (B, C) HK-2 cells transfected with control-siRNA or TLR4-siRNA were incubated with 5.5 or $30 \mathrm{mM}$ glucose for $30 \mathrm{~min}$ (B) or $24 \mathrm{~h}$ (C). Nuclear proteins were immunoblotted with an anti-p65 antibody. Histone $\mathrm{H} 3$ was used as a loading control of nuclear protein $(n=5)(B)$. Whole-cell lysates were immunoblotted with an anti-TGF- $\beta 1$ antibody $(n=3)(C) .\left({ }^{*} p<0.05\right.$ compared with control-siRNA and 5.5 $\mathrm{mM}$ glucose, ${ }^{* *} \mathrm{p}<0.05$ as compared with control-siRNA and $30 \mathrm{mM}$ glucose).

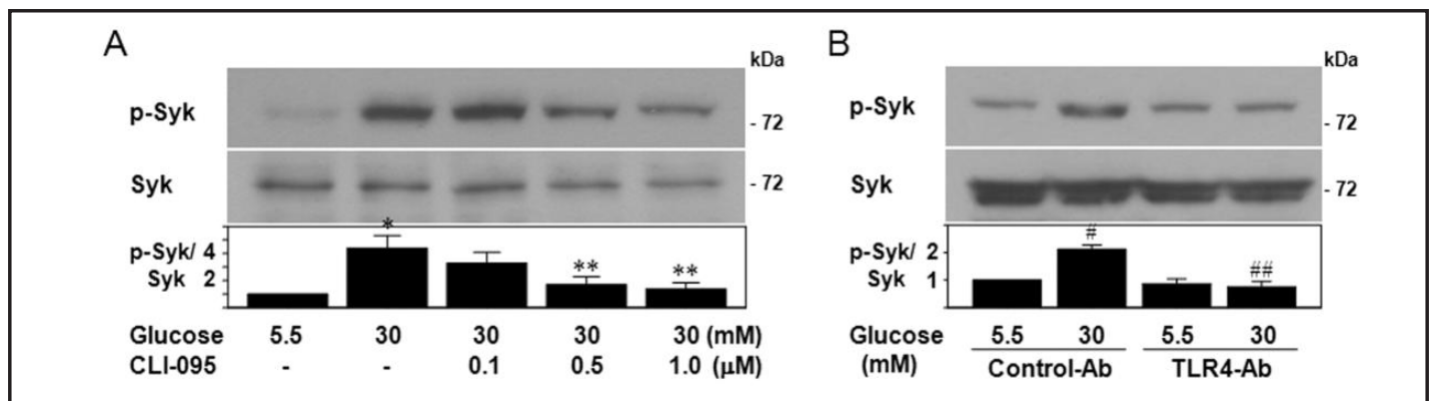

Fig. 3. Inhibition of TLR4 prevents high glucose-induced Syk activation. HK-2 cells preincubated with or without TLR4 inhibitor (CLI-095) (A) or TLR4-neutralizing antibody (5 $\mu \mathrm{g} / \mathrm{ml}$ ) (B) for $30 \mathrm{~min}$ were incubated with $30 \mathrm{mM}$ glucose for $10 \mathrm{~min}$. Whole-cell lysates were immunoblotted with an anti-phospho-Syk antibody. Thereafter, the membranes were stripped and reprobed with an anti-Syk antibody. $\left(n=5,{ }^{*} p<0.05\right.$ compared with $5.5 \mathrm{mM}$ glucose, ${ }^{* *} \mathrm{p}<0.05$ as compared with $30 \mathrm{mM}$ glucose; $\mathrm{n}=3$, ${ }^{*} \mathrm{p}<0.05$ compared with control $\mathrm{Ab}$ and $5.5 \mathrm{mM}$ glucose, ${ }^{\# \#} \mathrm{p}<0.05$ as compared with control $\mathrm{Ab}$ and $30 \mathrm{mM}$ glucose).

The role of TLR4 in high glucose-induced Syk activation was further confirmed by using a TLR4 antibody. Preincubation of HK-2 cells with neutralizing anti-TLR4 antibody ( $5 \mu \mathrm{g} / \mathrm{ml})$ attenuated high glucose-induced Tyr525 phosphorylation of Syk (Fig. 3B). 


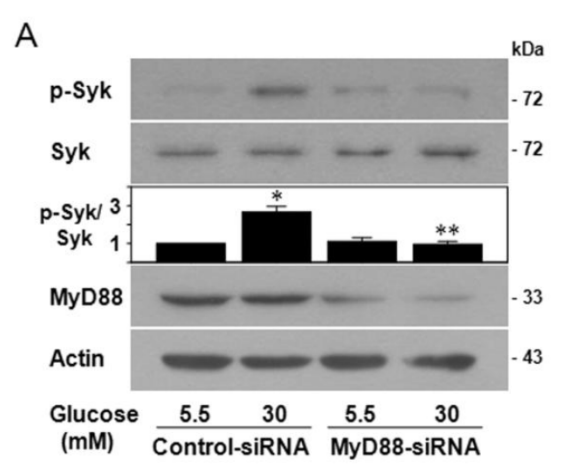

B

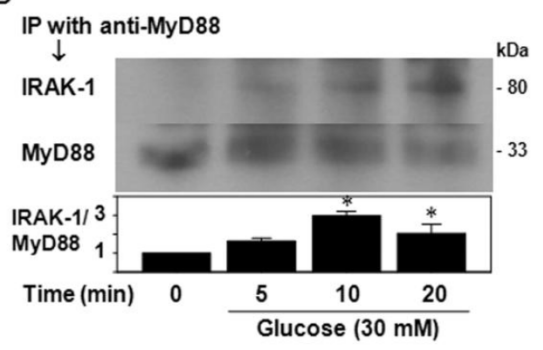

C IP with anti-TLR4

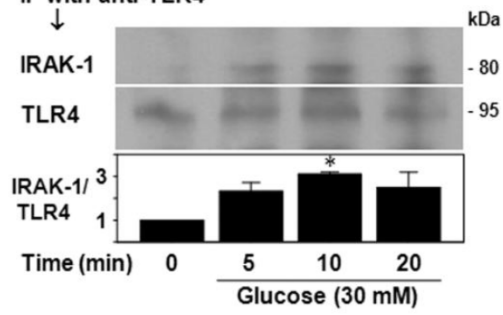

Fig. 4. Involvement of MyD88 in high glucose-induced Syk activation. (A) Depletion of MyD88 attenuates high glucose-induced Syk activation. HK-2 cells transfected with control-siRNA or MyD88-siRNA were incubated with 5.5 or $30 \mathrm{mM}$ glucose for $10 \mathrm{~min}$. Whole-cell lysates were immunoblotted with an anti-phospho-Syk antibody (upper panel) or an anti-MyD88 antibody (lower panel). Thereafter, the membranes were stripped and reprobed with an anti-Syk or anti-actin antibody, respectively. (n=4, *p $<0.05$ compared with control-siRNA and $5.5 \mathrm{mM}$ glucose, ${ }^{* *} \mathrm{p}<0.05$ as compared with control-siRNA and $30 \mathrm{mM}$ glucose) (B, C) High glucose stimulates the binding of IRAK-1 to MyD88 and TLR4. HK-2 cells were incubated with 30 mM glucose for 5, 10 or $20 \mathrm{~min}$. Whole-cell lysates were immunoprecipitated with an antibody to MyD88 (n=6) (B) or TLR4 $(\mathrm{n}=4)(\mathrm{C})$, and then immunoblotted with an anti-IRAK-1 antibody. $\left({ }^{*} \mathrm{p}<0.05\right.$ compared with control).

Fig. 5. Syk is associated with TLR4. HK-2 cells were stimulated with $30 \mathrm{mM}$ glucose for 5,10 or $20 \mathrm{~min}$. Whole-cell lysates were immunoprecipitated with an antibody to TLR4, and then immunoblotted with an anti-phospho-Syk antibody. Thereafter, the membranes were stripped and reprobed with an anti-Syk antibody. (n=4, *p $<0.05$ compared with control).

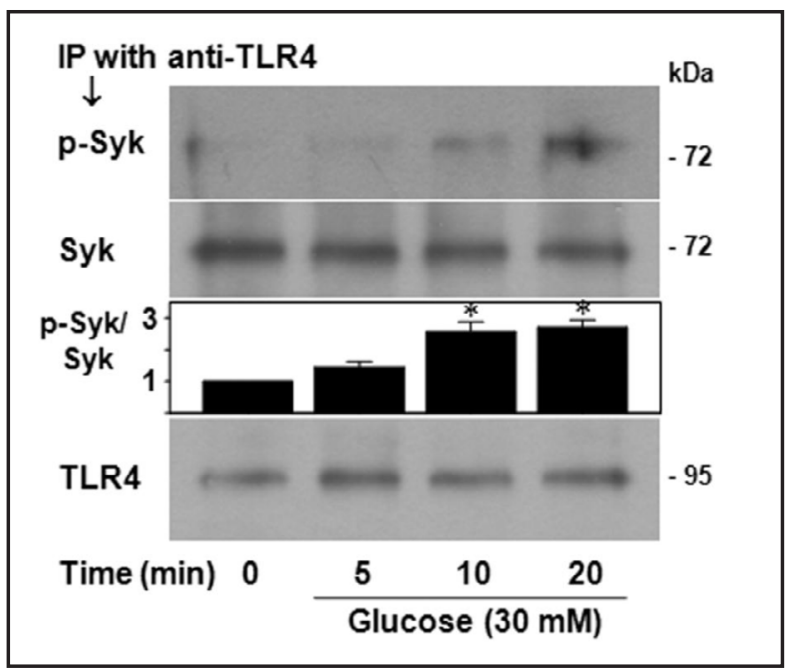

Involvement of MyD88 in high glucose-induced Syk activation

Since MyD88 is an adaptor protein for TLR4, and transmits the signal of activated TLR4, we examined whether MyD88 is involved in high glucose-induced Syk activation by knockdown of endogenous MyD88 using siRNA. Depletion of MyD88 resulted in a significant inhibition of high glucose-induced Syk activation (Fig. 4A). 

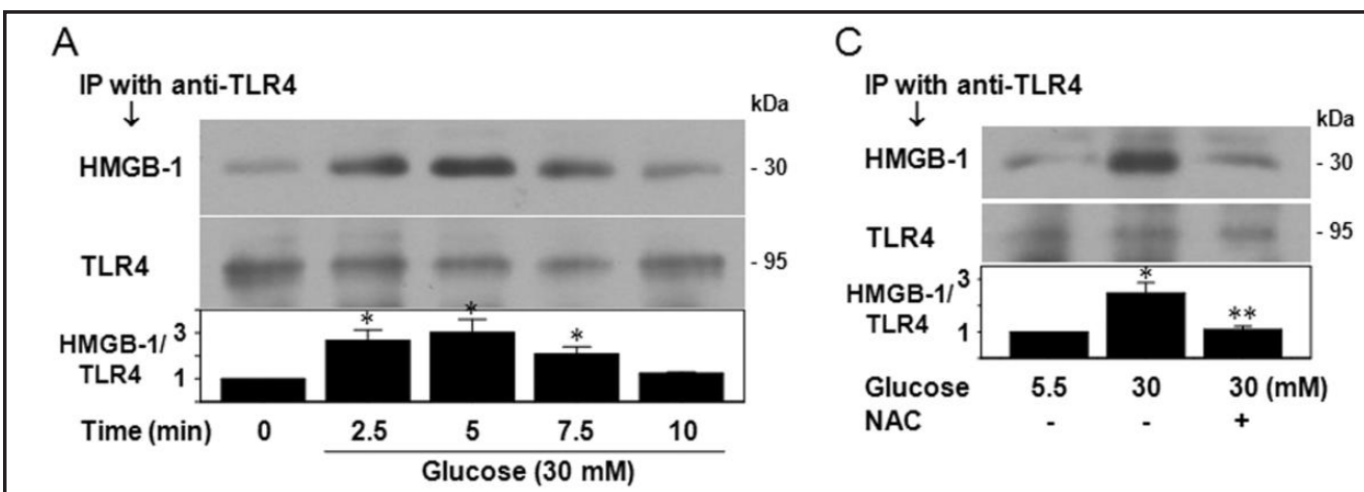

B
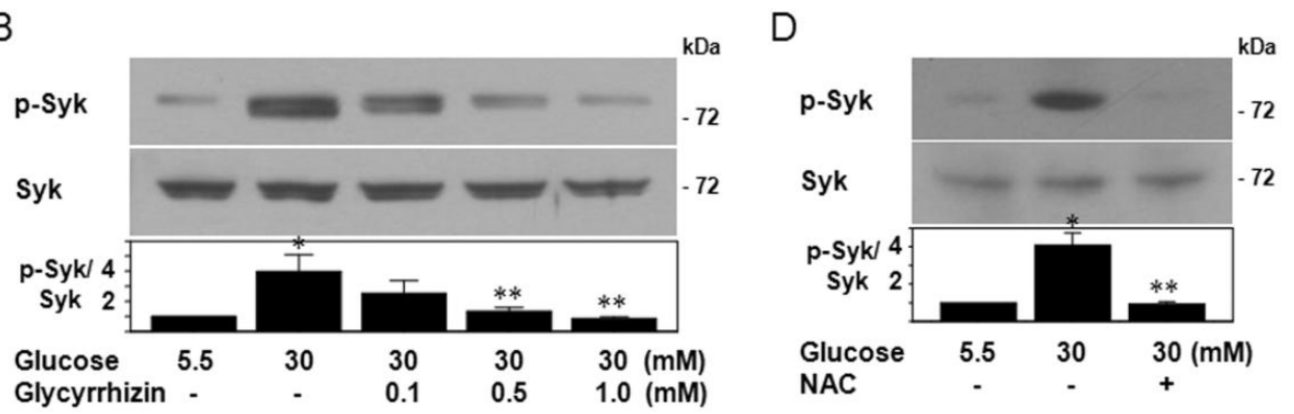

Fig. 6. HMGB-1 in high glucose-induced TLR4 activation. (A) High glucose increases the binding of HMGB-1 to TLR4. HK-2 cells were incubated with $30 \mathrm{mM}$ glucose for $0,2.5,5,7.5$ or $10 \mathrm{~min}$. Whole-cell lysates were immunoprecipitated with an antibody to TLR4, and then immunoblotted with an anti-HMGB-1 antibody. ( $\mathrm{n}=4,{ }^{*} \mathrm{p}<0.05$ compared with control) (B) HMGB-1 inhibitor attenuates high glucose-induced Syk activation. HK-2 cells were incubated for $10 \mathrm{~min}$ with $30 \mathrm{mM}$ glucose and different concentrations of glycyrrhizin. Whole-cell lysates were immunoblotted with an anti-phospho-Syk antibody. Thereafter, the membranes were stripped and reprobed with an anti-Syk antibody. $\left(n=4{ }^{*} \mathrm{p}<0.05\right.$ compared with $5.5 \mathrm{mM}$ glucose, **p $<0.05$ as compared with $30 \mathrm{mM}$ glucose) (C) N-acetylcysteine reduces high glucose-induced association of HMGB-1 with TLR4. HK-2 cells preincubated with or without N-acetylcysteine ( $5 \mathrm{mM}$ ) for 30 min were incubated with $30 \mathrm{mM}$ glucose for $5 \mathrm{~min}$. Whole-cell lysates were immunoprecipitated with an antibody to TLR4, and then immunoblotted with an anti-HMGB-1 antibody. ( $\mathrm{n}=5,{ }^{*} \mathrm{p}<0.05$ compared with $5.5 \mathrm{mM}$ glucose, **p $<0.05$ as compared with $30 \mathrm{mM}$ glucose) (D) $\mathrm{N}$-acetylcysteine inhibits high glucose-induced Syk activation. HK-2 cells preincubated with or without $\mathrm{N}$-acetylcysteine $(5 \mathrm{mM})$ for 30 min were incubated with $30 \mathrm{mM}$ glucose for $10 \mathrm{~min}$. Whole-cell lysates were immunoblotted with an anti-phospho-Syk antibody. Thereafter, the membranes were stripped and reprobed with an anti-Syk antibody. ( $n=5,{ }^{*} \mathrm{p}<0.05$ compared with 5.5 $\mathrm{mM}$ glucose, ${ }^{* *} \mathrm{p}<0.05$ as compared with $30 \mathrm{mM}$ glucose).

We also tested whether high glucose treatment is able to activate TLR4-MyD88 axis by measuring the recruitment of IRAK-1. HK-2 cells were incubated with $30 \mathrm{mM}$ glucose for 0 , 5, 10 or $20 \mathrm{~min}$, and whole cell lysates were immunoprecipitated with an antibody against MyD88 or TLR4 and then the immune complexes were subjected to immunoblot analysis with an antibody against IRAK-1. High glucose treatment rapidly increased the bindings of IRAK-1 to MyD88 and TLR4, as shown in Fig. 4B and 4C.

\section{Syk is associated with TLR4}

Next, we explored the interaction between TLR4 and Syk. HK- 2 cells were incubated with $30 \mathrm{mM}$ glucose for $0,5,10$ or $20 \mathrm{~min}$. Cell lysates were immunoprecipitated with an antibody against TLR4, and then the immune complexes were subjected to immunoblot analysis with an antibody against p-Syk and Syk. As shown in Fig. 5, Syk was co-immunoprecipitated with TLR4. High glucose did not increase the amount of Syk co-immunoprecipitated with TLR4, but increased phosphorylation of Syk bound to TLR4.

\section{KARGER}


Fig. 7. Schematic representation of the proposed signaling pathway of TLR4/Syk activation in high glucose stimulated HK-2 cells. Syk is constitutively associated with TLR4. Upon stimulation with high glucose, reactive oxygen species are produced and induce extracellular release of HMGB-1. HMGB-1 binds to TLR4 and activates it, which in turn leads to activation of Syk that is associated with TLR4. Activation of Syk leads to NF$\kappa \mathrm{B}$ activation and finally TGF-1 gene transcription. Abbreviations: HMGB-1, high-mobility group box-1; MD-2, myeloid differentiation factor 2 ; NF- $\kappa \mathrm{B}$, nuclear factor$\kappa \mathrm{B}$; Syk, spleen tyrosine kinase; TLR4, toll-like receptor 4 ; TGF- $\beta 1$, transforming growth factor- $\beta 1$.

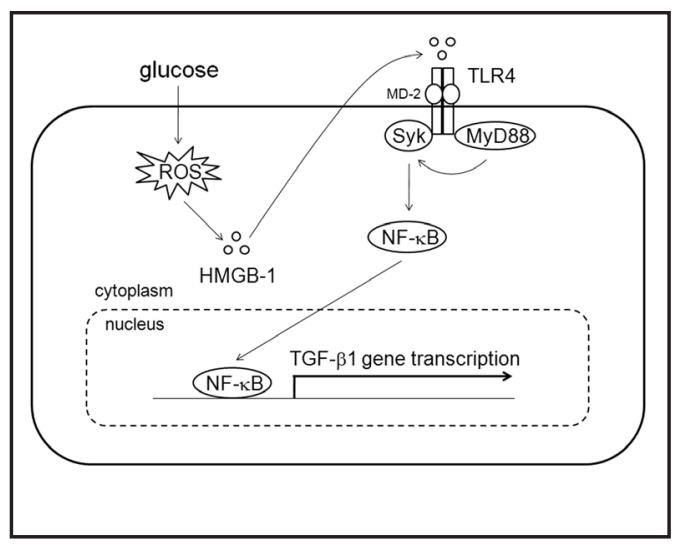

\section{HMGB-1 in high glucose-induced TLR4 activation}

HMGB-1 is an endogenous activator of TLR4. We further explored whether HMGB-1 is involved in the activation of high glucose-induced TLR4 activation. HK-2 cells were incubated with $30 \mathrm{mM}$ glucose for $0,2.5,5,7.5$ or $10 \mathrm{~min}$, and cell lysates were immunoprecipitated with an antibody against TLR4, and then the immune complexes were subjected to immunoblot analysis with an antibody against HMGB-1. As shown in Fig. 6A, high glucose increased the amount of HMGB-1 that was co-immunoprecipitated with TLR4. On the other hand, inhibition of HMGB-1 by glycyrrhizin suppressed high glucose-induced Syk activation (Fig. 6B). The association of HMGB-1 with TLR4 after high glucose treatment was reduced by pretreatment with $\mathrm{N}$-acetylcysteine (Fig. 6C). Consistent with it, high glucose-induced Syk activation was inhibited by $\mathrm{N}$-acetylcysteine (Fig. 6D).

\section{Discussion}

Our previous studies $[4,5]$ have shown that Syk is rapidly activated by high glucose and transmits high glucose signal leading to gene transcriptions of CCL2 and TGF- $\beta 1$, which could be implicated in the development of diabetic nephropathy. The pathway from high glucose to Syk activation, however, remains to be elucidated. Our data in the present study suggest the signal pathway as follows; Syk is constitutively associated with TLR4. High glucose induces an immediate extracellular release of HMGB-1 which binds and activates TLR4, leading to Syk activation.

In animal studies of diabetic nephropathy, inhibition of TLR4 conferred protective effects on albuminuria, renal dysfunction, renal cortical NF- $\kappa B$ activation, tubular CCL2 expression, and interstitial macrophage infiltration [12]. In vitro studies also showed that high glucose stimulates TLR4 gene transcription and its protein expression, while depletion of TLR4 resulted in reduction of NF- $\mathrm{KB}$ activation and resulting in downregulation of IL-6 and CCL2 expressions [11]. However, it is not known whether TLR4 participates in high glucose signal transduction before its expression is increased. Incubation of HK-2 cells in high glucose activates Syk within $10 \mathrm{~min}$. In the present study, depletion TLR4 by transfection of TLR4siRNA attenuated high glucose-induced Syk activation and the downstream events including NF- $\kappa$ B p 65 nuclear translocation and TGF- $\beta 1$ production. In addition, preincubation of HK- 2 cells with TLR4 inhibitor (CLI-095) or TLR4-neutralizing antibody attenuated high glucoseinduced Syk activation. MyD88 is an adaptor molecule of TLR4, and engagement of LPS to TLR4 induces the binding of IRAK-1 to MyD88, which transmits the LPS signal. Like LPS, high glucose rapidly induced the association of IRAK-1 to MyD88 and thereby to TLR4. These findings indicate that TLR4 is rapidly activated after high glucose stimulation and transmits the signal. 


\section{Cellular Physiology Cell Physiol Biochem 2015;35:2309-2319 \begin{tabular}{ll|l} 
and Biochemistry Published online: April 13, 2015 & $\begin{array}{l}\text { C) 2015 S. Karger AG, Basel } \\
\text { www.karger.com/cpb }\end{array}$ \\
\hline
\end{tabular} \\ Yang et al.: TLR4/Syk in High Glucose Signal Transduction}

The role of Syk in intracellular signal transductions has been studied mainly in hematopoietic cells [1], and Syk was found to form a complex with TLR4 in neutrophils, monocytes and macrophages [7-9]. The N-terminal SH2 domain of Syk was suggested to interact with the cytoplasmic TIR domain of TLR4 [18]. This interaction is constitutive because Syk is associated with TLR4 in the absence of any stimuli. Stimulation with LPS in neutrophil was shown to further increase Syk binding to TLR4, with an increase in Syk activation [7]. In another study of monocyte, however, LPS did not increase the association between TLR4 and Syk [8], but induced the activation of Syk that is constitutively bound to TLR4. In the present study, Syk in HK-2 cells was co-immunoprecipitated with TLR4 in the resting state and thus Syk was constitutively associated with TLR4, as in the hematopoietic cells. High glucose did not increase the association of Syk to TLR4, but stimulated the activation of Syk co-immunoprecipitated with TLR4. Since Syk mediates high glucose signal leading to TGF- $\beta 1$ production and the activation of Syk was dependent on TLR4 as noted above, Syk bound to TLR4 seems to play a key role in the signal transduction of TLR4 that is activated by high glucose.

TLR4 signaling has been known to occur via two pathways; MyD88-dependent pathway (TIRAP-MyD88-IRAK) and MyD88-independent pathway (TRAM-TRIF-IRF3) [6]. Depending on the nature of stimuli, TLR4 signaling occurs via recruitment of different adaptor molecules to the cytoplasmic domain of the receptor, thus resulting in different cellular responses [19]. In macrophage, minimally oxidized low-density lipoprotein stimulated intracellular reactive oxygen species (ROS) generation via TLR4 and Syk activation, while depletion of MyD88 did not alter the ROS production. Thus, Syk was suggested to be another TLR4 adaptor molecule [9]. In case of HK-2 cells in the present study, depletion of MyD88 attenuated high glucoseinduced Syk activation. Along with the finding of the increased binding of IRAK-1 to MyD88 implying activation of MyD88, it suggests that MyD88 is also implicated in high glucoseinduced Syk activation.

Another question addressed in this study was how TLR4 is rapidly activated by high glucose. TLRs interact with endogenous substances, i.e., damage-associated molecular pattern molecules (DAMPs), as well as pathogen-associated molecular patterns [10]. DAMPs are released from cells in response to injury, infection, or other inflammatory stimuli and activate TLRs and thereby mediate systemic inflammatory responses [20]. HMGB-1 is a nonhistone nuclear protein that is expressed constitutively in mammalian cells and contributes to stabilization of nucleosomes, DNA repair and recombination, and transcription [21]. Under stress conditions, it is released from the cells and functions as a DAMP. Recent studies have shown that high glucose induces HMGB-1 translocation from nucleus to cytosol and extracellular release $[22,23]$. ROS are also known to induce extracellular release of HMGB-1 [24]. Because it is well-known that high glucose induces ROS generation, it may cause extracellular release of HMGB-1 through ROS. In these studies [22, 24], the increase of HMGB-1 in culture media was significant only after several hours of exposure to high glucose or ROS, as measured by ELISA or western blot. The delayed release of HMGB-1 does not account for the early activation of TLR4 shown in the present study. However, our data suggests that HMGB-1 is released immediately. HMGB-1 was co-immunoprecipitated with TLR4, and the amount of HMGB-1 bound to TLR4 began to increase as early as 2.5 min after exposure to high glucose with a peak at $5 \mathrm{~min}$ and then decreased. In contrast, glycyrrhizin, an inhibitor of HMGB-1, suppressed high glucose-induced Syk activation when it was added simultaneously with high glucose stimulation. Thus, HMGB-1 seems to be rapidly released by high glucose, and binds and activates TLR4 on the cell surface. Reduction of the amount of HMGB-1 co-immunoprecipitated with TLR4 by N-acetylcysteine, an antioxidant, suggests that the release of HMGB-1 is dependent on ROS produced by high glucose stimulation.

In summary, Syk in HK-2 cells is constitutively associated with TLR4 as in hematopoietic cells. Upon stimulation with high glucose, TLR4 is immediately activated by HMGB-1, which in turn leads to activation of Syk that is associated with TLR4 (Fig. 7). In this way, TLR4/Syk complex plays a key role in high glucose signal transduction. 


\section{Acknowledgement}

This study was supported by a grant (02-200) from the Asan Institute for Life Sciences (Seoul, Korea). We thank Kim Jin Ju and Moon Soo Young for excellent technical assistance.

\section{Disclosure Statement}

The authors declare no potential conflict of interest.

\section{References}

1 Sada K, Takano T, Yanagi S, Yamamura H: Structure and function of Syk protein-tyrosine kinase. J Biochem 2001;130:177-186.

2 Patterson H, Nibbs R, McInnes I, Siebert S: Protein kinase inhibitors in the treatment of inflammatory and autoimmune diseases. Clin Exp Immunol 2014;176:1-10.

3 Yanagi S, Inatome R, Takano T, Yamamura H: Syk expression and novel function in a wide variety of tissues. Biochem Biophys Res Commun 2001;288:495-498.

4 Yang WS, Seo JW, Han NJ, Choi J, Lee KU, Ahn H, Lee SK, Park SK: High glucose-induced NF-кB activation occurs via tyrosine phosphorylation of $\mathrm{I} \kappa \mathrm{B} \alpha$ in human glomerular endothelial cells: involvement of Syk tyrosine kinase. Am J Physiol Renal Physiol 2008;294:F1065-F1075.

$5 \quad$ Yang WS, Chang JW, Han NJ, Lee SK, Park SK: Spleen tyrosine kinase mediates high glucose-induced transforming growth factor- $\beta 1$ up-regulation in proximal tubular epithelial cells. Exp Cell Res 2012;318:1867-1876.

6 Kumar H, Kawai T, Akira S: Toll-like receptors and innate immunity. Biochem Biophys Res Commun 2009;388:621-625.

7 Arndt PG, Suzuki N, Avdi NJ, Malcolm KC, Worthen GS: Lipopolysaccharide-induced c-Jun NH2-terminal kinase activation in human neutrophils: role of phosphatidylinositol 3-kinase and Syk-mediated pathways. J Biol Chem 2004;279:10883-10891.

8 Chaudhary A, Fresquez TM, Naranjo MJ: Tyrosine kinase Syk associates with toll-like receptor 4 and regulates signaling in human monocytic cells. Immunol Cell Biol 2007;85:249-256.

9 Bae YS, Lee JH, Choi SH, Kim S, Almazan F, Witztum JL, Miller YI: Macrophages generate reactive oxygen species in response to minimally oxidized low-density lipoprotein: toll-like receptor 4- and spleen tyrosine kinase-dependent activation of NADPH oxidase 2. Circ Res 2009;104:210-218.

10 Yu L, Wang L, Chen S: Endogenous toll-like receptor ligands and their biological significance. J Cell Mol Med 2010;14:2592-2603.

11 Lin M, Yiu WH, Wu HJ, Chan LY, Leung JC, Au WS, Chan KW, Lai KN, Tang SC: Toll-like receptor 4 promotes tubular inflammation in diabetic nephropathy. J Am Soc Nephrol 2012;23:86-102.

12 Lin M, Yiu WH, Li RX, Wu HJ, Wong DW, Chan LY, Leung JC, Lai KN, Tang SC: The TLR4 antagonist CRX-526 protects against advanced diabetic nephropathy. Kidney Int 2013;83:887-900.

13 Cha JJ, Hyun YY, Lee MH, Kim JE, Nam DH, Song HK, Kang YS, Lee JE, Kim HW, Han JY, Cha DR: Renal protective effects of toll-like receptor 4 signaling blockade in type 2 diabetic mice. Endocrinology 2013;154:2144-2155.

14 Kaur H, Chien A, Jialal I: Hyperglycemia induces Toll like receptor 4 expression and activity in mouse mesangial cells: relevance to diabetic nephropathy. Am J Physiol Renal Physiol 2012;303:F1145-F1150.

15 Zhang J, Billingsley ML, Kincaid RL, Siraganian RP: Phosphorylation of Syk activation loop tyrosines is essential for Syk function. An in vivo study using a specific anti-Syk activation loop phosphotyrosine antibody. J Biol Chem 2000;275:35442-35447.

16 Garg A, Aggarwal BB: Nuclear transcription factor $-\kappa B$ as a target for cancer drug development. Leukemia 2002;16:1053-1068. 


\section{Cellular Physiology Cell Physiol Biochem 2015;35:2309-2319}

and Biochemistry

17 Matsunaga N, Tsuchimori N, Matsumoto T, Ii M: TAK-242 (resatorvid), a small-molecule inhibitor of Tolllike receptor (TLR) 4 signaling, binds selectively to TLR4 and interferes with interactions between TLR4 and its adaptor molecules. Mol Pharmacol 2011;79:34-41.

18 Choi SH, Harkewicz R, Lee JH, Boullier A, Almazan F, Li AC, Witztum JL, Bae YS, Miller YI: Lipoprotein accumulation in macrophages via toll-like receptor-4-dependent fluid phase uptake. Circ Res 2009;104:1355-1363.

19 Miller YI, Choi SH, Wiesner P, Bae YS: The SYK side of TLR4: signalling mechanisms in response to LPS and minimally oxidized LDL. Br J Pharmacol 2012;167:990-999.

20 Van Crombruggen K, Jacob F, Zhang N, Bachert C: Damage-associated molecular patterns and their receptors in upper airway pathologies. Cell Mol Life Sci 2013;70:4307-4321.

21 Yanai $\mathrm{H}$, Ban T, Taniguchi T: High-mobility group box family of proteins: ligand and sensor for innate immunity. Trends Immunol 2012;33:633-640.

22 Wang Y, Shan J, Yang W, Zheng H, Xue S: High mobility group box 1 (HMGB1) mediates high-glucoseinduced calcification in vascular smooth muscle cells of saphenous veins. Inflammation 2013;36:15921604.

23 Dasu MR, Devaraj S, Park S, Jialal I: Increased toll-like receptor (TLR) activation and TLR ligands in recently diagnosed type 2 diabetic subjects. Diabetes Care 2010;33:861-868.

24 Tang D, Shi Y, Kang R, Li T, Xiao W, Wang H, Xiao X: Hydrogen peroxide stimulates macrophages and monocytes to actively release HMGB1. J Leukoc Biol 2007;81:741-747. 